\title{
Mind the gap: optimising medical registrar rota provision
}

\author{
Author: Katharine Fairbairn
}

\section{Aims}

To determine if adjustment in the medical registrar weekend cover would reduce the number of rota gaps and subsequent locum spend.

\section{Methods}

As RCP chief registrar at an acute general hospital, I was tasked with assessing the effect of implementing a change to the medical registrar on-call rota; incorporating the general medicine and elderly care weekend registrar cover into a single rota. Previously, medical and elderly care patients had separate registrars for weekend day cover.

As with other similar sized hospitals in our region, the trust has been affected significantly by reduced numbers of higher medical trainees entering elderly care and general internal medicine. As such we had seen increasing numbers of medical on-call rota gaps, with the obvious implications for patient safety. To cover these gaps, the trust often has to turn to locum cover, frequently at enhanced rates, with the added financial implications. There was further pressure on our trust as the number of filled posts was scheduled to fall further following the registrar rotations in February and March 2018.

A 6-month period prior to the change was analysed for this project and post-implementation data is still being collected.

\section{Results}

Prior to the planned introduction of the combined rota in February 2018, there were 11.5 full-time equivalent (FTE) registrars for an equivalent of 14 positions ( $82 \%$ fill rate). This was due to reduce to 8.5 FTE (61\% fill rate) following the February/March 2018 rotation.

Prior to the new rota implementation, there was a mean of 8.7 unfilled medical on-call registrar shifts per month (median 7.5). The cost of covering these locum shifts at standard registrar rate would equate to $£ 6,264$ per month, not accounting for enhanced rates for antisocial hours or locum agencies.

Based on the number of rota gaps above, there would have been a predicted 19.1 shifts per month requiring cover if we were to remain with the previous rota pattern of two registrars on weekend days, at a cost of $£ 13,752$ per month.

Author: Royal College of Physicians chief registrar, Poole Hospital NHS Trust, Poole, UK

\section{Conclusion}

The implementation of the new combined rota was essential to ensure sufficient senior medical cover at weekends, both for patients and junior trainees. To date, there appears to have been a reduction in the number of persistent rota gaps requiring cover, with predicted reduction in financial cost.

\section{Conflict of interest statement}

None declared. 